\title{
Colpopexia Sacroespinhal: Análise de sua Aplicação em Portadoras de Prolapso Uterovaginal e de Cúpula Vaginal Pós-Histerectomia
}

\author{
Sacrospinous Colpopexy: Analysis of its Use in Patients with Uterovaginal \\ and Vaginal Vault Prolapse after Histerectomy \\ Octacílio Figueirêdo Netto ${ }^{1}$, Octacílio Figueirêdo ${ }^{2}$, José Rafael Macéa ${ }^{3}$, \\ Roberto Adelino de Almeida Prado ${ }^{4}$
}

\section{RESUMO}

Objetivo: analisar os resultados de uma variante técnica de colpopexia sacroespinhal para tratamento cirúrgico do prolapso de cúpula vaginal, e também como medida adjuvante nos casos de prolapso uterovaginal total, visando facilitar o procedimento.

Métodos: quarenta e seis pacientes foram operadas e acompanhadas por periodo de 12 a 44 meses, com média de 32 meses. Vinte e três pacientes apresentavam prolapso de cúpula vaginal (GCúpula) e 23 eram portadoras de prolapso uterovaginal (GÚtero). O critério de inclusão foi a presença de prolapso sintomático grau III ou IV, segundo a classificação proposta pela Sociedade Internacional de Continência. Foram excluidas pacientes portadoras de prolapso de menor grau. A média de idade das pacientes foi semelhante: 67,0 anos no GCúpula e 67,5 anos no GÚtero. O índice de massa corpórea médio também foi semelhante, sendo de $27,4 \mathrm{~kg} / \mathrm{m}^{2}$ no GCúpula e de $25,6 \mathrm{~kg} / \mathrm{m}^{2}$ no Gútero. A paridade variou de 0 a 13 partos nas pacientes do GCúpula, com média de 4,4 partos, e de 1 a 13 partos nas pacientes do GÚtero, com média de 6,2 partos. Entre as 23 pacientes do GCúpula, oito (34,7\%) já haviam sido submetidas à cirurgia para correção do problema, sem sucesso. Os resultados obtidos em ambos os grupos foram analisados e comparados. O método utilizado obedece a princípios anatômicos bem definidos, e difere da técnica original pelo emprego de portaagulha curvo orientado de cima para baixo para transfixar o ligamento sacroespinhal direito com suturas sob visão direta, aproximadamente dois $\mathrm{cm}$ medialmente à espinha isquiática, minimizando assim o risco de lesão dos vasos e nervo pudendo.

Resultados: a média de duração da cirurgia foi de 90,0 minutos no GCúpula e 119,5 minutos no GÚtero $(p<0,05)$. Ocorreram três transfusões sanguíneas, uma no GCúpulae duas no GÚtero, não havendo lesões vesicais, retais, ureterais ou óbitos em nenhum dos grupos. A incidência e o tipo de complicações pós-operatórias foram semelhantes nos dois grupos estudados: infecção urinária, granuloma, retenção urinária, neuropatia transitória, dor na nádega e transfusão sanguínea. O comprimento vaginal médio após a operação foi de 7,6 cm nas pacientes do GCúpula e de 7,3 cm nas do GÚtero ( $p>0,05)$. O resultado anatômico dos compartimentos vaginais apical, anterior e posterior foi satisfatório em mais de $90 \%$ das pacientes de ambos os grupos. O resultado funcional também foi semelhante entre os grupos, sendo que das pacientes com vida sexual ativa, apenas uma (7,7\%) do GCúpula e duas (13,3\%) do GÚtero queixaram-se de dispareunia após a cirurgia. Não foi verificada associação entre idade, paridade, obesidade e os resultados anatomofuncionais.

Conclusão: a análise dos dados obtidos demonstra que esta variante de colpopexia sacroespinhal é tecnicamente simples, segura e eficaz, fornecendo resultados semelhantes nos dois grupos de pacientes estudados.

PALAVRAS-CHAVE: Prolapso genital. Prolapso de cúpula vaginal. Prolapso uterovaginal. Colpopexia sacroespinhal. Histerectomia. Cirurgia: complicações. 
Introdução

O prolapso uterovaginal e de cúpula vaginal pós-histerectomia representam desafios para o cirurgião ginecológico. Em 1997, Olson et al. ${ }^{1}$ verificaram que a chance de uma mulher ser submetida à cirurgia para correção de prolapso genital durante a vida é de $11 \%$, sendo quase um terço das operações realizadas para correção de recidivas. Estes dados indicam não só a complexidade dos defeitos do assoalho pélvico com os quais o cirurgião ginecológico se depara, como também a necessidade de conhecimento adequado da anatomia da pelve feminina e fisiopatologia do prolapso, permitindo que a operação seja realizada de maneira segura e eficaz. O tratamento cirúrgico do prolapso genital quase sempre implica a correção simultânea de múltiplos defeitos do assoalho pélvico, e atualmente preconizase a correção sítio-específica individualizada de cada um destes defeitos, na tentativa de melhorar o resultado a longo prazo.

A posição normal dos órgãos pélvicos resulta da interação entre músculos e tecido conjuntivo. Há, portanto, associação entre estruturas de suspensão (complexo ligamentar uterossacro/cardinal) e de sustentação (diafragma pélvico) que, quando alterada, favorece o desenvolvimento do prolapso genital. Na posição em pé, o terço superior da vagina apresenta orientação quase horizontal e repousa sobre o diafragma pélvico, representado principalmente pelo músculo elevador do ânus $^{2}$. Segundo DeLancey ${ }^{3}$, as condensações ligamentares de tecido conjuntivo estabilizam a vagina em três diferentes niveis. No nível I, os ligamentos uterossacros e cardinais suspendem o colo uterino e terço superior da vagina sobre o músculo elevador do ânus, em posição distante do hiato genital. No nível II, o terço médio da vagina é mantido na sua posição normal pela inserção lateral da fáscia endopélvica no arco tendíneo da fáscia pélvica. No nível III, ocorre fusão do terço inferior da vagina com estruturas que a circundam. Quando as estruturas que estabilizam a va-

\footnotetext{
${ }^{1}$ Departamento de Ginecologia e Obstetrícia da Universidade Estadual de Londrina - PR

${ }^{2}$ Endofemina - Ginecologia e Obstetrícia, Londrina - PR ${ }^{3}$ Departamento de Morfologia da Faculdade de Ciências Médicas da Santa Casa - SP

${ }^{4}$ Departamento de Obstetrícia e Ginecologia da FCM da Santa Casa - SP

Correspondência:

Octacílio Figueirêdo Netto

Rua Belo Horizonte, 1445 apto.1601

86020-060 - Londrina - PR

Fone: (43) 3323-3682 - Fax: (43) 3356-3076

e-mail: octaciliof@uol.com.br
}

gina estão íntegras, a bexiga, uretra, colo uterino e reto são mantidos na posição anatômica normal. Com o aumento da pressão intra-abdominal a vagina é comprimida no sentido ântero-posterior contra os músculos elevadores do ânus, que ao mesmo tempo se contraem ${ }^{4}$. O prolapso uterovaginal total é acompanhado de eversão completa da vagina, e ocorre quando a integridade dos ligamentos uterossacros e cardinais (nivel I) é insuficiente para posicionar o ápice vaginal sobre o diafragma pélvico. O enfraquecimento destes ligamentos normalmente está associado ao alargamento do hiato genital, com perda da sustentação e conseqüente descida do útero ${ }^{5}$. Já no prolapso de cúpula pós-histerectomia, a ausência de fáscia endopélvica na altura do ápice vaginal favorece o surgimento de enterocele através do espaço compreendido entre as fáscias pubocervical e retovaginal $^{3}$. Assim, o prolapso de cúpula vaginal é considerado a única hérnia genital verdadeira, já que o peritônio se encontra em contato direto com a mucosa vaginal.

O prolapso genital pode ser tratado de forma conservadora ou cirurgicamente. Mais de quarenta técnicas já foram descritas para seu tratamento, o que demonstra que nenhuma delas pode ser considerada ideal. Os procedimentos cirúrgicos são classificados como obliterativos ou reconstrutivos, podendo ser realizados por via vaginal, abdominal e, mais recentemente, por via laparoscópica. A colpopexia sacroespinhal foi inicialmente descrita na Europa em meados do século passado e posteriormente difundida nos Estados Unidos ${ }^{6}$. Apesar de ter sido originalmente proposta para tratamento do prolapso de cúpula vaginal pós-histerectomia, também tem sido sugerido seu emprego durante a histerectomia vaginal para tratamento do prolapso uterovaginal, pois nestes casos o encurtamento dos paramétrios durante a histerectomia pode ser insuficiente para a suspensão adequada da cúpula ${ }^{7}$. Assim, atualmente a maioria dos trabalhos preconiza a utilização da colpopexia sacroespinhal tanto na correção do prolapso de cúpula, como de forma adjuvante no tratamento do prolapso uterovaginal ${ }^{8,9}$.

Embora seja técnica cirúrgica consagrada mundialmente, a colpopexia sacroespinhal ainda é pouco utilizada no Brasil, e as principais razões para isto são: idéia equivocada de que a cirurgia é muito difícil e perigosa e percepção errônea de que são necessários intrumentos dispendiosos para sua realização. Além disto, o número de cirurgiões com experiência suficiente para ensiná-la durante a residência médica é limitado. Neste trabalho propomos uma variante técnica de colpopexia sacroespinhal com o intuito de minimizar estas li- 
mitações, principalmente o uso de instrumentos especiais e o receio de ocorrência de hemorragia incoercível decorrente de lesões vasculares. Os resultados de sua aplicação tanto em pacientes portadoras de prolapso de cúpula vaginal como naquelas com prolapso uterovaginal são comparados, visando reforçar o valor de sua aplicação neste último grupo de pacientes.

\section{Pacientes e Métodos}

Entre junho de 1999 e março de 2002, 46 pacientes foram submetidas à colpopexia sacroespinhal e acompanhadas durante período médio de 32 meses, variando de 12 a 44 meses. Vinte e três pacientes apresentavam prolapso de cúpula vaginal (GCúpula), ao passo que outras 23 eram portadoras de prolapso uterovaginal (GÚtero). O critério de inclusão foi a presença de prolapso sintomático grau III (exteriorização do útero/cúpula vaginal além do intróito vaginal) ou IV (eversão completa da vagina), segundo a classificação proposta pela Sociedade Internacional de Continência ${ }^{10}$. Foram excluídas pacientes portadoras de prolapso de menor grau.

A idade das pacientes variou de 49 a 81 anos no GCúpula, com média de 67,0 anos e de 42 a 86 anos no GÚtero, com média de 67,5 anos. O índice de massa corpórea (IMC) variou de 20,0 a $32,9 \mathrm{~kg}$ / $\mathrm{m}^{2}$ nas pacientes do GCúpula, com média de 27,4 $\mathrm{kg} / \mathrm{m}^{2}$ e de 19,5 a $35,7 \mathrm{~kg} / \mathrm{m}^{2}$ nas do Gútero, com média de $25,6 \mathrm{~kg} / \mathrm{m}^{2}$. A paridade variou de 0 a 13 partos nas pacientes do GCúpula, com média de 4,4 partos, e de 1 a 13 partos nas pacientes do GÚtero, com média de 6,2 partos. Entre as 23 pacientes do GCúpula, $8(34,7 \%)$ já haviam sido submetidas à cirurgia para correção do problema, sem sucesso.

As seguintes variáveis foram estudadas e comparadas entre os dois grupos: duração da cirurgia, complicações intra-operatórias, complicações pós-operatórias, comprimento da vagina após a cirurgia, resultado anatômico dos compartimentos vaginais apical, anterior e posterior e resultado funcional no pós-operatório. O estudo foi aprovado pelo Comitê de Ética em Pesquisa da Universidade Estadual de Londrina. A análise estatística dos resultados foi realizada por meio do teste $t$ de Student para amostras pareadas e teste do $\chi^{2}$ para análise comparativa. Diferença significativa foi verificada quando se obteve $\mathrm{p} \leq 0,05$.

A técnica de colpopexia sacroespinhal é a mesma, independente da realização ou não de histerectomia concomitante. No prolapso uterovaginal, inicialmente o útero é removido de maneira habitual, sem preocupação com encurtamento dos paramétrios ou sua aproximação na linha mediana, já que não serão utilizados para a suspensão da cúpula vaginal. Quando existe cistocele e/ou incontinência urinária concomitante, a abordagem do compartimento vaginal anterior precede a colpopexia, sendo a mucosa anterior suturada de maneira contínua com categute 0 simples. Realiza-se então incisão transversal interessando pele e mucosa do intróito vaginal, seguida da abertura longitudinal da mucosa posterior até o ápice vaginal, com união das incisões das mucosas anterior e posterior. Quase que invariavelmente nota-se a presença de enterocele, a qual é tratada pela dissecção do saco herniário com tesoura e sua sutura em bolsa com fio de poliglactina 1 , seguido da ressecção do excesso de peritônio. Após dissecção ampla do espaço retovaginal, penetra-se no espaço pararretal direito de maneira romba com os dedos indicador e médio. A espinha isquiática direita é palpada e os dedos são direcionados póstero-medialmente, identificando-se o ligamento sacroespinhal, uma estrutura firme que se estende de cada uma das espinhas isquiáticas até a porção inferior do sacro e cóccix. Suas referências anatômicas são a espinha isquiática lateralmente, a borda lateral do sacro medialmente, a face póstero-superior do músculo piriforme e a margem ântero-inferior do músculo ileococcígeo. Às vezes o ligamento sacroespinhal se funde com o músculo coccígeo (Figura 1). Três válvulas de Breisky são então introduzidas no espaço pararretal para ampliá-lo e possibilitar a visualização direta e transfixação do ligamento sacroespinhal.

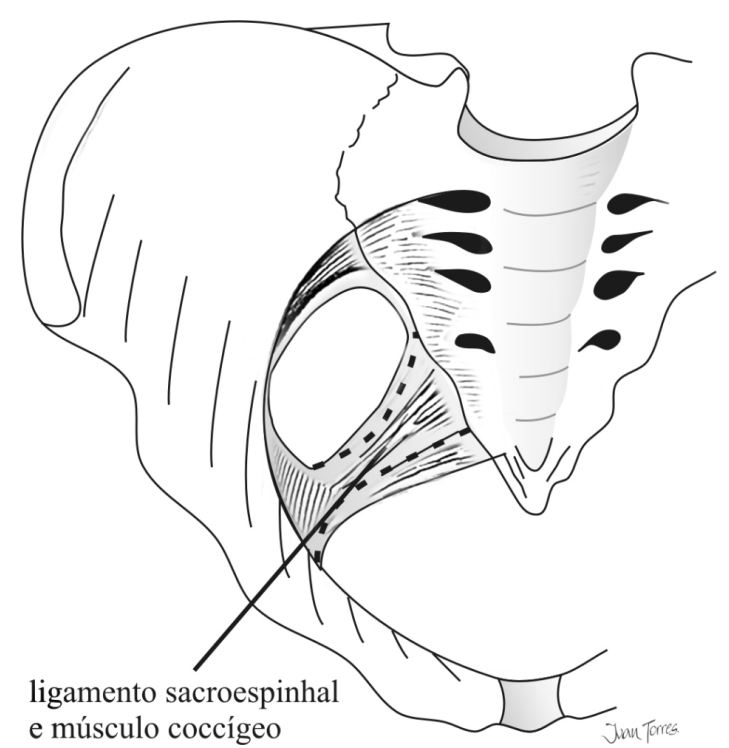

Figura 1 - Relação anatômica entre o ligamento sacroespinhal e o músculo coccígeo. 
A variante técnica aqui proposta consiste em transfixar o ligamento com dois fios de poliglactina 1 , cerca de $2 \mathrm{~cm}$ medialmente à espinha, utilizando uma técnica original na qual o porta-agulha curvo de Heaney é orientado de cima para baixo e da direita para a esquerda da paciente $^{11}$ (Figura 2). Após a transfixação do ligamento, a agulha permanece presa ao portaagulha, sendo então removida com porta-agulha de Mayo ao mesmo tempo em que se libera o porta-agulha de Heaney. O excesso de mucosa é ressecado e a mesma é suturada de modo contínuo ancorado com categute 0 simples até o ápice vaginal. $\mathrm{O}$ ápice vaginal ainda prolapsado é então transfixado com os dois fios passados no ligamento sacroespinhal direito, sendo que na metade lateral da mucosa vaginal utiliza-se o fio mais lateral, e na metade medial o fio mais medial.

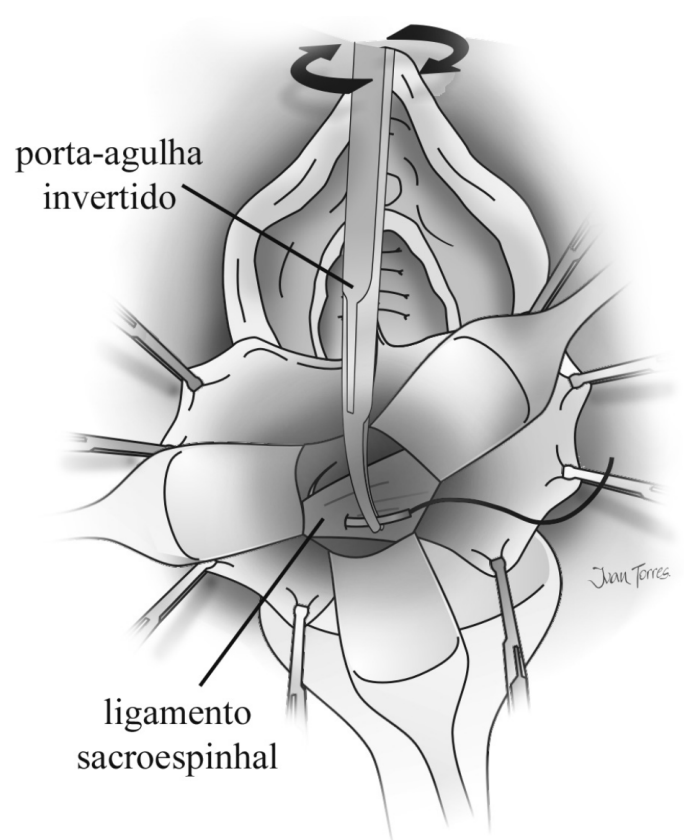

Figura 2 - Transfixação do ligamento sacroespinhal.

Neste momento, a mucosa vaginal é suturada até aproximadamente o terço inferior da parede posterior e as suturas colocadas no ligamento sacroespinhal direito são então amarradas, colocando a cúpula vaginal em aposição ao ligamento, e novamente sobre o músculo elevador do ânus (Figura 3). Após a correção de eventual retocele e estreitamento do hiato genital com a aproximação do músculo elevador do ânus, o centro tendíneo do períneo é reconstituído e a pele suturada com categute 0 simples.

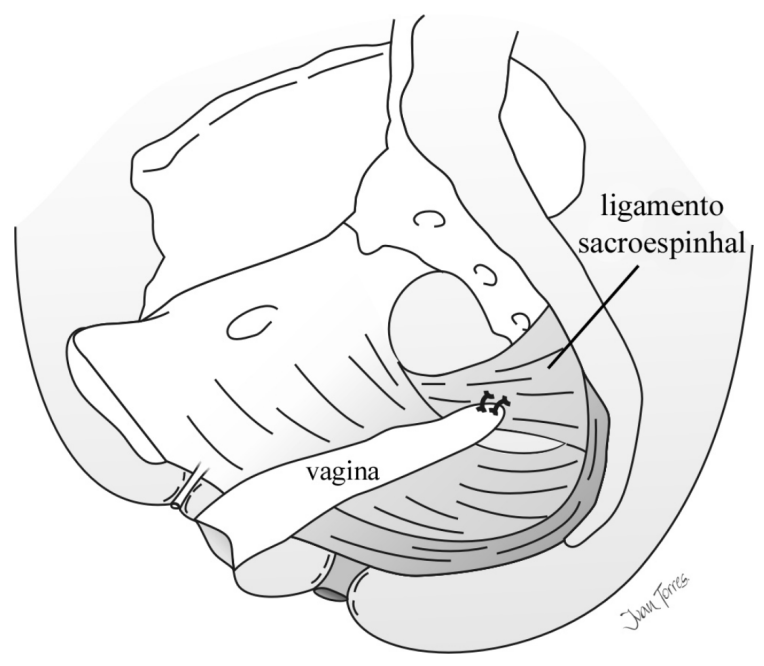

Figura 3 - Fixação da cúpula vaginal no ligamento sacroespinhal.

\section{Resultados}

A duração da cirurgia variou de 40 a 150 minutos nas pacientes do GCúpula, com média de 90 minutos, e de 90 a 240 minutos nas pacientes do GÚtero, com média de 119,5 minutos. A diferença entre as médias de duração da cirurgia entre os dois grupos foi estatisticamente significativa $(\mathrm{p}<0,05)$.

Em relação às complicações intra-operatórias, ocorreram três transfusões sanguíneas, uma no GCúpula e duas no GÚtero, não havendo lesões vesicais, retais, ureterais ou óbitos em nenhum dos grupos. A Tabela 1 ilustra a incidência e o tipo de complicação pós-operatória em cada um dos grupos estudados, não tendo sido observada diferença estatisticamente significativa entre os mesmos. O comprimento vaginal médio após a cirurgia foi de 7,6 $₫ 1,6 \mathrm{~cm})$ nas pacientes do GCúpula, e de 7,3 $( \pm 1,1 \mathrm{~cm})$ nas do GÚtero, não havendo diferença significativa entre os dois grupos.

Tabela 1 - Complicações pós-operatórias nos dois grupos de pacientes estudados.

\begin{tabular}{lcc}
\hline Tipo de complicação & GCúpula (\%) & GÚtero (\%) \\
\hline Infecção urinária & $2(8,7)$ & - \\
Granuloma & $2(8,7)$ & - \\
Retenção urinária & $1(4,3)$ & $1(4,3)$ \\
Neuropatia transitória & - & $2(8,7)$ \\
Dor na nádega & $1(4,3)$ & $2(8,7)$ \\
Infecção urinária + retenção & - & $2(8,7)$ \\
Transfusão sanguínea & $1(4,3)$ & $1(4,3)$ \\
Total & $23(100)$ & $23(100)$ \\
\hline
\end{tabular}


A Tabela 2 mostra o resultado anatômico dos compartimentos vaginais apical, anterior e posterior nos dois grupos estudados, ou seja, o grau de prolapso eventualmente encontrado nas pacientes após seguimento mínimo de 12 meses.

Tabela 2 - Resultado anatômico e pós-operatório dos compartimentos vaginais apical, anterior e posterior nos dois grupos de pacientes estudados.

\begin{tabular}{lcccc}
\hline Grupo & $\begin{array}{c}\text { Grau de } \\
\text { Prolapso }^{10}\end{array}$ & Apical (\%) & Anterior (\%) & Posterior (\%) \\
\hline GCúpula & - & $21(91,3)$ & $21(91,3)$ & $21(91,3)$ \\
& I & $1(4,3)$ & $1(4,3)$ & $1(4,3)$ \\
& II & $1(4,3)$ & - & - \\
& III & 0 & $1(4,3)$ & $1(4,3)$ \\
GÚtero & - & $21(91,3)$ & $21(91,3)$ & $21(91,3)$ \\
& I & $2(8,7)$ & $2(8,7)$ & $2(8,7)$ \\
\hline
\end{tabular}

Nota: Não foi observado prolapso grau II, III ou IV no seguimento de pacientes do GÚtero, nem prolapso grau IV nas pacientes do GCúpula.

Quanto ao resultado funcional da colpopexia sacroespinhal, 13 pacientes $(56,5 \%)$ no GCúpula e 15 pacientes $(65,2 \%)$ no GÚtero tinham vida sexual ativa. Uma paciente $(7,7 \%)$ do GCúpula e duas pacientes $(13,3 \%)$ do GÚtero se queixaram de dispareunia após a cirurgia. Não foi verificada associação entre a idade, paridade, obesidade e os resultados anatomofuncionais da cirurgia.

\section{Discussão}

O prolapso uterovaginal e de cúpula vaginal às vezes causa pouco desconforto para as pacientes, podendo ser tratado conservadoramente. Entretanto, quando sintomático, o prolapso genital constitui um dos problemas de mais difícil resolução em cirurgia ginecológica. Entre as diversas alternativas de tratamento cirúrgico do prolapso genital, a colpopexia sacroespinhal vem sendo utilizada com sucesso há vários anos, apresentando algumas vantagens em relação a outros métodos: preservação da vagina, importante não só na manutenção da função sexual, como também da feminilidade e imagem corporal; menor agressão cirúrgica com o uso da via vaginal em relação à via abdominal, principalmente levando-se em conta a fragilidade das pacientes; maior facilidade para correção de outras distopias freqüentemente associadas; eficácia e segurança comprovadas; e baixo custo ${ }^{12,13}$.

A agulha de Deschamps foi o instrumento de escolha para realização da cirurgia até o final da década de 80. Posteriormente, Miyazaki ${ }^{14}$ descreveu a utilização do Miya hook para facilitar a passagem dos pontos no ligamento sacroespinhal. Com a consolidação da colpopexia sacroespinhal como técnica bem estabelecida, as empresas de material cirúrgico passaram a desenvolver instrumentos, muitos deles descartáveis, para a realização do procedimento, como o Capio e o Endo Stitch, dispositivos que apreendem o fio automaticamente após a transfixação do ligamento ${ }^{15,16}$. Nossa experiência com os diversos instrumentos desenvolvidos para realização da colpopexia sacroespinhal foi negativa, seja porque sua extremidade distal relativamente romba dificulta a transfixação do ligamento, às vezes esgarçando as fibras do mesmo, ou devido ao tamanho excessivo das agulhas, prejudicando o controle da extensão da transfixação do ligamento. Além disso, muitas vezes a quantidade de ligamento transfixado é bem inferior ao que julgamos adequado para fixação eficiente da cúpula vaginal ${ }^{17}$. Some-se a isso o alto custo dos instrumentos descartáveis e a dificuldade para a aquisição de alguns instrumentos, restringindo sua aplicação prática no nosso meio.

A variante técnica aqui proposta obedece a preceitos anatômicos que visam facilitar e aumentar a segurança da cirurgia. O ligamento sacroespinhal é acessado através do espaço pararretal, área relativamente avascular, exceto pequenos ramos da artéria retal média que suprem o tecido adventício deste espaço. Os vasos pudendos encontram-se a aproximadamente $0,5 \mathrm{~cm}$ da espinha isquiática, na borda lateral do ligamento sacroespinhal. Portanto, o cirurgião deve passar os pontos cerca de $2 \mathrm{~cm}$ medialmente à espinha, e as suturas devem ser realizadas através do ligamento, e não ao redor do mesmo ${ }^{18}$. Ao transfixarmos o ligamento sacroespinhal com o porta-agulha orientado de cima para baixo, automaticamente o ponto é passado da posição lateral para medial, minimizando o risco de lesão de estruturas nobres situadas próximo à espinha isquiática. É importante enfatizar a necessidade de se remover a agulha com outro porta-agulha, que neste caso deve ser preferencialmente um porta-agulha reto convencional. A remoção da agulha com outro porta-agulha evita que a mesma se perca no espaço pararretal. Normalmente se utiliza o ligamento sacroespinhal direito, já que a maioria dos cirurgiões são destros e a dissecção romba do espaço pararretal com os dedos indicador e médio da mão direita é mais fácil, do mesmo modo que é mais fácil para os destros a realização da episiotomia médio-lateral direita.

Além da diversidade de instrumentos que 
podem ser utilizados para transfixação do ligamento sacroespinhal, também foram descritas algumas modificações no conceito original do método. A colpopexia sacroespinhal pode ser realizada bilateralmente, e a indicação mais comum para o emprego de ambos os ligamentos é o prolapso de cúpula vaginal recidivado ${ }^{19}$. Entretanto, estudos recentes têm demonstrado que a colpopexia bilateral, embora viável, não reduz a incidência de distopias subseqüentes ${ }^{20}$. Recentemente tem sido proposta a realização da cirurgia através do espaço paravesical (colpopexia anterior), e não pararretal (colpopexia posterior), como forma de restaurar o eixo vaginal normal e reduzir a incidência de cistocele subseqüente. Entretanto, alguns autores notaram maior incidência de retocele pós-operatória nestas mulheres ${ }^{21}$.

Os grupos GCúpula e GÚtero eram semelhantes quanto à idade e obesidade. A segurança e eficácia da colpopexia sacroespinhal em pacientes muito idosas também foi comprovada por Kaminsky et al. ${ }^{22}$, dado relevante visto que a maioria das pacientes que apresentam prolapso genital total pertence a uma faixa etária avançada. Nieminen e Heinonen ${ }^{9}$ verificaram inclusive que a cirurgia é segura em mulheres acima de 80 anos de idade. As mulheres obesas são particularmente predispostas ao desenvolvimento de prolapso genital devido ao aumento constante da pressão intra-abdominal. Nestas pacientes em particular, ressalta-se a importância de se adotarem técnicas de prevenção do prolapso de cúpula durante a histerectomia, como a culdoplastia de McCall e o estreitamento do hiato genital alargado por meio da aproximação do músculo elevador do ânus.

O emprego profilático da colpopexia sacroespinhal para prevenção do prolapso de cúpula vaginal após histerectomia vaginal realizada em paciente portadora de prolapso uterovaginal ainda é tema controvertido. Nestes casos, a simples culdoplastia é insuficiente para uma suspensão adequada da cúpula vaginal, já que esta se encontra evertida e exteriorizada, tornando necessária a fixação da cúpula numa estrutura mais firme. No nosso meio, Marana et al. ${ }^{23}$ realizaram estudo para determinar se somente a histerectomia vaginal associada à colporrafia anterior e posterior representava alternativa eficaz a longo prazo no tratamento do prolapso uterovaginal total. Entre as 47 pacientes acompanhadas, apenas duas $(4,3 \%)$ não apresentaram algum grau de prolapso dos órgãos pélvicos após seguimento médio de cinco anos. Nossa conduta está em concordância com a recomendação proposta em estudos recentes que preconizam a realização da colpopexia sacroespinhal como medida adjuvante nestes $\operatorname{casos}^{24,25}$.
O tempo de cirurgia das 46 pacientes estudadas variou de 40 a 240 minutos, semelhante ao encontrado no estudo de 156 pacientes realizado por Pasley ${ }^{26}$, no qual variou de 37 a 234 minutos. A duração da cirurgia foi em média 29 minutos mais rápida nas pacientes portadoras de prolapso de cúpula vaginal, diferença estatisticamente significativa em relação às pacientes operadas por prolapso uterovaginal total. Chapin ${ }^{27}$ constatou diferença semelhante, de 22 minutos, nas pacientes submetidas à histerectomia vaginal concomitante. O maior tempo cirúrgico deste último grupo explica-se não só pela realização da histerectomia, como também pela maior extensão da reconstrução do assoalho pélvico nestes casos, a qual quase invariavelmente inclui a correção de defeitos de todos os compartimentos vaginais: anterior, apical e posterior.

Quanto às complicações intra-operatórias, as três transfusões sanguíneas $(6,5 \%)$ observadas nas 46 pacientes estudadas foram decorrentes da perda sanguínea resultante da grande área de dissecção, e não de hemorragia resultante da dissecção do espaço pararretal ou passagem dos pontos no ligamento sacroespinhal. Outros autores encontraram resultados semelhantes, como Penalver et al. ${ }^{28}$, que relataram sete transfusões $(4,4 \%)$ em 160 pacientes. A incidência e o tipo de complicações pós-operatórias observadas neste estudo estão em concordância com a literatura ${ }^{29}$, bem como a semelhança observada entre o GÚtero e o GCúpula ${ }^{24,25}$. A dor na nádega é complicação que pode eventualmente requerer a remoção da sutura quando se utiliza fio inabsorvivel ${ }^{30}$.

O comprimento vaginal médio encontrado em ambos os grupos após a cirurgia foi semelhante $(7,6 \mathrm{~cm}$ no GCúpula e $7,3 \mathrm{~cm}$ no GÚtero). Given et al. ${ }^{31}$ avaliaram o comprimento vaginal após a colpopexia sacroespinhal, encontrando resultados semelhantes, ou seja, $8,2 \mathrm{~cm}$ nas pacientes com prolapso de cúpula vaginal e $8,5 \mathrm{~cm}$ naquelas com prolapso uterovaginal. O resultado anatômico da colpopexia sacroespinhal foi semelhante tanto para mulheres portadoras de prolapso de cúpula vaginal como para aquelas com prolapso uterovaginal total, dado também constatado por outros autores $^{9}$. Nossa taxa de sucesso de 91,3\% na suspensão da cúpula vaginal está em concordância com os achados de Pasley ${ }^{26}$, o qual obteve êxito em $94,4 \%$ de suas pacientes. O discreto desvio lateral da vagina praticamente desaparece após alguns meses. Em extensa revisão da literatura, Sze e Karram ${ }^{13}$ compilaram 22 estudos envolvendo um total de 1229 procedimentos, sendo 1062 (86\%) pacientes disponíveis para seguimento no pós-operatório durante período que va- 
riou de um mês a 11 anos. Em relação ao resultado anatômico após a colpopexia sacroespinhal, 109 (18\%) das 1062 pacientes avaliadas apresentaram recidiva do prolapso genital, incluindo 81 defeitos no compartimento vaginal anterior, 32 recidivas apicais, 24 defeitos no compatimento posterior e 56 defeitos em locais não especificados ou múltiplos. Foi necessário tratamento cirúrgico adicional em sete das 81 pacientes com cistocele recorrente, 20 das 32 pacientes com prolapso de cúpula vaginal e quatro das 24 pacientes com retocele.

Nossos achados quanto à função sexual após a cirurgia estão de acordo com os da literatura, havendo queixa de dispareunia em uma paciente $(4,3 \%)$ do GCúpula e em duas pacientes $(8,7 \%)$ do GÚtero. Holley et al. ${ }^{32}$ acompanharam 36 pacientes durante 15 a 79 meses e verificaram três casos de dispareunia devido à constrição da vagina. As outras pacientes sexualmente ativas relataram melhora ou manutenção da função sexual. Richter e Albrich ${ }^{12}$ relataram que cinco (6\%) de 81 pacientes não mantinham relações após a cirurgia devido à estenose da vagina. Given et al. ${ }^{31}$ verificaram que a colpopexia sacroespinhal não reduziu o comprimento vaginal nem interferiu na função sexual em um grupo de 59 mulheres submetidas à colpopexia. Nas pacientes com vida sexual ativa é imprescindivel que se tenha cuidado redobrado para evitar estenose vaginal decorrente da aproximação excessiva do músculo elevador do ânus na linha média. Além disso, é importante que os dois pontos no ligamento sacroespinhal sejam passados a pelo menos $2 \mathrm{~cm}$ um do outro, evitando assim o afunilamento do ápice vaginal.

A análise dos nossos resultados permite afirmar que este método simplificado de colpopexia sacroespinhal é eficaz e acarreta baixa morbidade intra e pós-operatória, proporcionando resultados anatomofuncionais satisfatórios tanto em pacientes portadoras de prolapso uterovaginal como naquelas com prolapso de cúpula vaginal. Esperamos que isto estimule maior utilização deste procedimento no nosso meio, não só no tratamento do prolapso de cúpula pós-histerectomia, mas também como medida adjuvante nos casos de prolapso uterovaginal total. Eliminando as incertezas que restringem o emprego desta cirurgia no arsenal terapêutico do cirurgião ginecológico, esperamos contribuir para que ele alcance os objetivos da reconstrução pélvica: alívio dos sintomas, restauração da anatomia e manutenção das funções vesical, intestinal e sexual normais.

\section{ABSTRACT}

Purpose: to analyze the results of a technical alternative to perform sacrospinous colpopexy for the treatment of vault prolapse after hysterectomy, and also as an additional facilitating procedure in cases of total uterovaginal prolapse.

Methods: forty-six patients underwent hysterectomy and were followed-up for 12 to 44 months, with an average of 32 months. Twenty-three of them presented vaginal vault prolapse (GVault), and 23 had total uterovaginal prolapse (GUterus). The inclusion criterion was the presence of symptomatic prolapse grade III or IV according to the classification proposed by the International Continence Society. Patients presenting lower grade prolapse were excluded. The average age of the patients was similar: 67.0 years in GVault and 67.5 years in GUterus. Average body mass index was also similar: $27.4 \mathrm{~kg} / \mathrm{m}^{2}$ in GVault and 25.6 $\mathrm{kg} / \mathrm{m}^{2}$ in GUterus. Deliveries varied from 0 to 13 in GVault (average: 4.4 deliveries), and from 1 to 13 in GUterus (average: 6.2 deliveries). Among the 23 patients in GVault, eight had undergone previous surgical repair without success. The results obtained in both groups were analyzed and compared. The used method takes into account wellknown anatomical principles, and differs from the original technique by using a curved needle holder oriented upside down to place sutures through the right sacrospinous ligament under direct vision, approximately $2 \mathrm{~cm}$ medially to the ischial spine, thus minimizing the risk of injury to the pudendal vessels and nerve.

Results: average duration of the surgery was $90.0 \mathrm{~min}$ in GVault and 119.5 min in GUterus, a statistically significant difference $(p<0.05)$. Three blood transfusions were needed, one in GVault and two in GUterus. There was no bladder, rectal or ureteral injury nor death in any of the groups. The incidence and type of postoperative complications were similar in the two groups, and included urinary infection, granuloma, urinary retention, transient neuropathy, buttock pain and blood transfusion. Average vaginal length after the operation was $7.6 \mathrm{~cm}$ in GVault and $7.3 \mathrm{~cm}$ in GUterus $(p>0.05)$. The anatomical result of the apical, anterior and posterior vaginal compartments was satisfactory in more than $90 \%$ of the patients of both groups. The functional result was also similar in both groups, and among the sexually active patients, only one (7.7\%) in GVault and two (13.3\%) in GUterus complained of dyspareunia after the surgery. There was no association between age, parity, obesity, and the anatomical and functional results.

Conclusion: analysis of the obtained data demonstrates that this modification of sacrospinous colpopexy is technically simple, safe and effective, providing similar results in both groups of the studied patients.

KEYWORDS: Genital prolapse. Vaginal vault prolapse. Sacrospinous colpopexy. Uterovaginal prolapse. Hysterectomy. Surgery: complications. 


\section{Referências}

1. Olsen AL, Smith VJ, Bergstrom JO, Colling JC, Clark AL. Epidemiology of surgically managed pelvic organ prolapse and urinary incontinence. Obstet Gynecol 1997; 89:501-6.

2. Macéa JR. Diafragma pélvico. Femina 1995; 23:695-7.

3. DeLancey JO. Anatomic aspects of vaginal eversion after hysterectomy. Am J Obstet Gynecol 1992; 166:1717-24.

4. Funt MI, Thompson JD, Birch H. Normal vaginal axis. South Med J 1978; 71:1534-5, 1552.

5. Nichols DH. Defeitos do compartimento central. In: Rock JA, Thompson JD, editores. Te Linde Ginecologia Operatória. $8^{a}$ ed. Rio de Janeiro: Guanabara Koogan; 1999. p. 792-812.

6. Randall CL, Nichols DH. Surgical treatment of vaginal inversion. Obstet Gynecol 1971; 38:327-32.

7. Cruikshank SH, Cox DW. Sacrospinous fixation at the time of transvaginal hysterectomy. Am J Obstet Gynecol 1990; 162:1611-5.

8. Hoffman MS, Harris MS, Bouis PJ. Sacrospinous colpopexy in the management of uterovaginal prolapse. J Reprod Med 1996; 41:299-303.

9. Nieminen K, Heinonen PK. Sacrospinous ligament fixation for massive genital prolapse in women aged over 80 years. BJOG 2001; 108:817-21.

10.Bump RC, Mattiasson A, BO K, et al. The standardization of terminology of female pelvic organ prolapse and pelvic floor dysfunction. Am J Obstet Gynecol 1996; 175:10-7.

11.Figueirêdo ON, Figueirêdo O, Pereira RMA. Complicações intra e pós-operatórias. In: Figueirêdo ON, editor. Histerectomia Vaginal: novas perspectivas. $2^{\mathrm{a}}$ ed. Londrina: Midiograf; 2004. p. 222-8.

12.Richter K, Albrich W. Long-term results following fixation of the vagina on the sacrospinous ligament by the vaginal route (vaginaefixatio sacrospinalis vaginalis). Am J Obstet Gynecol 1981; 141:811-6.

13.Sze EH, Karram MM. Transvaginal repair of vault prolapse: a review. Obstet Gynecol 1997; 89:466-75.

14.Miyazaki FS. Miya Hook ligature carrier for sacrospinous ligament suspension. Gynecol Obstet 1987; 70:286-8.

15.Lind LR, Choe J, Bhatia NN. An in-line suturing device to simplify sacrospinous vaginal vault suspension. Obstet Gynecol 1997; 89:129-32.

16. Watson JD. Sacrospinous ligament colpopexy: new instrumentation applied to a standard gynecologic procedure. Obstet Gynecol 1996; 88:883-5.

17. Veronikis DK, Nichols DH. Ligature carrier specifically designed for transvaginal sacrospinous colpopexy. Obstet Gynecol 1997; 89:478-81.
18. Morley GW. Vaginal approach to treatment of vaginal vault eversion. Clin Obstet Gynecol 1993; 36:984-94.

19.Imparato E, Aspesi G, Rovetta E, Presti M. Surgical management and prevention of vaginal vault prolapse. Surg Gynecol Obstet 1992; 175:233-7.

20.Pohl JF, Frattarelli JL. Bilateral transvaginal sacrospinous colpopexy: preliminary experience. Am J Obstet Gynecol 1997; 177:1356-61.

21.Goldberg RP, Tomezsko JE, Winkler HA, Koduri S, Culligan PJ, Sand PK. Anterior or posterior sacrospinous vault suspension: long-term anatomic and functional evaluation. Obstet Gynecol 2001; 98:199-204.

22.Kaminsky PF, Sorosky JI, Pees RC, Podczaski ES. Correction of massive vaginal prolapse in an older population: a four-year experience at a rural tertiary care center. J Am Geriatr Soc 1993; 41:42-4.

23.Marana HR, Andrade JM, Marana RR, Matheus de Sala M, Philbert PM, Rodrigues R. Vaginal hysterectomy for correcting genital prolapse. Long-term evaluation. J Reprod Med 1999; 44:529-34.

24.Guner H, Noyan V, Tiras MB, Yidiz A, Yildirim M. Transvaginal sacrospinous colpopexy for marked uterovaginal and vault prolapse. Int $J$ Gynaecol Obstet 2001; 74:165-70.

25.Lantzsch T, Goepel C, Wolters M, Koelbl H, Methfessel HD. Sacrospinous ligament fixation for vaginal vault prolapse. Arch Gynecol Obstet 2001; 265:21-5.

26.Pasley WW. Sacrospinous suspension: a local practitioner's experience. Am J Obstet Gynecol 1995; 173:440-5.

27.Chapin DS. Teaching sacrospinous colpopexy. Am J Obstet Gynecol 1997; 177:1330-6.

28.Penalver M, Mekki Y, Lafferty H, Escobar M, Angioli R. Should sacrospinous ligament fixation for the management of pelvic suport defects be part of a residency program procedure? The University of Miami experience. Am J Obstet Gynecol 1998; 178:326-9.

29.Sauer HA, Klutke CG. Transvaginal sacrospinous ligament fixation for treatment of vaginal prolapse. J Urol 1995; 154:1008-12.

30.Alevizon SJ, Finan MA. Sacrospinous colpopexy: management of postoperative pudendal nerve entrapment. Obstet Gynecol 1996; 88:713-5.

31.Given FT Jr, Muhlendorf IK, Browning GM. Vaginal length and sexual function after colpopexy for complete uterovaginal eversion. Am J Obstet Gynecol 1993; 169:284-7.

32.Holley RL, Varner RE, Gleason BP, Apffel LA, Scott $\mathrm{S}$. Sexual function after sacrospinous ligament fixation for vaginal vault prolapse. J Reprod Med 1996; 41:355-8. 